\title{
Transition from Positive to Negative Photoconductance in Doped Hybrid Perovskite Semiconductors
}

Md Azimul Haque, Jin-Ling Li, Ahmed L. Abdelhady, Makhsud I. Saidaminov, Derya Baran, Osman M. Bakr, Su-Huai Wei*, Tom Wu*

M. A. Haque, Prof. D. Baran

King Abdullah University of Science and Technology (KAUST), Physical Science and Engineering Division, KAUST Solar Center (KSC), Thuwal 23955-6900, Saudi Arabia

Dr. A. L. Abdelhady, Dr. M. I. Saidaminov, Prof. O. M. Bakr

Division of Physical Sciences and Engineering, King Abdullah University of Science and Technology (KAUST), Thuwal 23955-6900, Kingdom of Saudi Arabia

J. Li, Prof. S.-H. Wei

Beijing Computational Science Research Center, Beijing 100193, China

suhuaiwei@csrc.ac.cn

Prof. T. Wu

School of Materials Science and Engineering, University of New South Wales (UNSW), Sydney, NSW 2052, Australia

tom.wu@unsw.edu.au

Keywords: Hybrid perovskites, doping; negative photoconductance, DX centers

Halide perovskites have been known to be photoconductive for more than half a century, and their efficient photocarrier generation gives rise to positive photoconductivity $(P P C)$. In this work, we report the discovery of negative photoconductivity (NPC) in hybrid perovskite $\mathrm{CH}_{3} \mathrm{NH}_{3} \mathrm{PbBr}_{3}$ after Bi doping. Transient photoconductivity measurements reveal a surprising bipolar behavior with a fast positive response followed by exponential negative photocurrent decay, resulting in an equilibrium photocurrent even below the dark level. The NPC effect in Bidoped $\mathrm{CH}_{3} \mathrm{NH}_{3} \mathrm{PbBr}_{3}$ is among the largest ones reported so far for semiconductors. We propose that the transition to negative photoconductance is related to the presence of DX-like centers in Bi-doped halide perovskites, similar to doped III-V and chalcopyrite semiconductors. Such photogenerated DX-like centers in the Bi-doped $\mathrm{CH}_{3} \mathrm{NH}_{3} \mathrm{PbBr}_{3}$ can trap mobile band electrons 


\section{WILEY-VCH}

and enhance charge recombination, thus reducing the conductivity. This mechanism is consistent with the observations of crossover from PPC to NPC as functions of temperature, composition and illumination. Our results underscore the importance of defect engineering for tuning the optoelectronic properties of halide perovskites.

Solution-processed organic-inorganic halide perovskites have recently attracted interest in the materials research community because of their intriguing physical properties and excellent photovoltaic performance. ${ }^{[1]}$ Different from common elemental and compound semiconductors, these hybrid materials are composed of organic groups embedded in networks of inorganic $\mathrm{Pb}-\mathrm{I}$ $(\mathrm{Br}, \mathrm{Cl})$ octahedra. These halide perovskites have been known to be photoconductive for six decades. ${ }^{[2]}$ However, only till recently, their extraordinary physical properties have not been pursued for technological advances. Apart from solar cells, photodetectors and LEDs, ${ }^{[3]}$ hybrid perovskites have been exploited for applications in X-ray detectors, ${ }^{[4]}$ gamma detectors, ${ }^{[5]}$ lasers $^{[6]}$ and memory devices. ${ }^{[7]}$ The recent demonstration of optical cooling of $23 \mathrm{~K}$ from room temperature in $\mathrm{CH}_{3} \mathrm{NH}_{3} \mathrm{PbI}_{3}\left(\mathrm{MAPbI}_{3}\right)$ suggests that the quality of such hybrid crystals is on par with the best inorganic semiconductors. ${ }^{[8]}$ It is noteworthy that most of the solar cells and optoelectronic devices based on hybrid perovskites till date have been fabricated using intrinsic perovskite layers without intentional doping. Recently, there have been some reports on doping or alloying hybrid perovskites with elements such as $\mathrm{Bi}, \mathrm{Mn}, \mathrm{Fe}, \mathrm{Co}, \mathrm{Ni}, \mathrm{Ag}$, and $\mathrm{Pd} .{ }^{[9]}$ However, there remain open questions regarding how doping impacts the physical properties and defect characteristics of hybrid perovskites.

Upon illumination, semiconductors usually show increased electrical conductivity due to the generation of photo-excited carriers. But in some unusual cases, conductivity decreases under 


\section{WILEY-VCH}

illumination, even below the thermal equilibrium level, which is termed as negative photoconductivity (NPC). Although NPC is often considered as anomalous, there have been reports on NPC phenomenon for more than one century in a wide variety of materials such as Co-doped $\mathrm{Si}^{[10]} \mathrm{Se}^{[11]} \mathrm{InAs},{ }^{[12]} \mathrm{Ag} / \mathrm{Au}$ nanoparticles, ${ }^{[13]} \gamma$-In $2 \mathrm{Se}_{3},{ }^{[14]} \mathrm{CNT},{ }^{[15]}$ and Bi-doped ZnSe. ${ }^{[16]}$ However, there has been no consensus regarding the mechanism of NPC. Factors such as enhanced majority carrier recombination rate,${ }^{[17]}$ and scattering of charge carriers ${ }^{[18]}$ have been evoked to account for NPC, but the reasons why NPC emerges in certain materials at certain conditions remain poorly understood. For example, in one early report, NPC was linked with an increase of polarized defect-rich regions under illumination. ${ }^{[19]}$ In another study on $\mathrm{Ag}_{2} \mathrm{~S}$ with the addition of $1-2 \% \mathrm{PbS}$, it was concluded that some illumination-activated defect states capture electrons from the conduction band, leading to the emergence of NPC. ${ }^{[20]}$ Although these mechanisms are different in their fundamental aspects, they all show some common basic properties of NPC such as narrowness of the energy range it is observed, slow kinetics of both positive and negative PC and that NPC can be triggered by increasing the recombination or trapping centers for photogenerated carriers within the system. ${ }^{[21]}$ A more precise model for the occurrence of NPC was proposed by Stokmann in 1955 suggesting increased majority carrier recombination rate being responsible for NPC ${ }^{[17]}$ This model is one of the most widely used mechanisms to explain NPC in various materials till date. In these early reports, the importance of impurity levels was recognized, but the identification and interaction between trapping defects and photo-excited carriers remains elusive. Furthermore, the investigation on NPC has not been extended so far beyond inorganic and carbon based materials. NPC based optoelectronics may find applications in photodetection, optical switching and nonvolatile memory in the future. ${ }^{[12,15}$, 


\section{WILEY-VCH}

The present work focuses on the surprisingly rich optoelectronic properties of Bi-doped hybrid perovskite $\mathrm{CH}_{3} \mathrm{NH}_{3} \mathrm{PbBr}_{3}\left(\mathrm{MAPbBr}_{3}\right)$. $\mathrm{Bi}$ has been one of the most favorable n-type dopant choices because it is adjacent to $\mathrm{Pb}$ in the periodic table with similar ionic sizes. Bi-doped $\mathrm{MAPbI}_{3}$ was investigated for applications in LED and optical amplification, ${ }^{[23]}$ and $\mathrm{Pb}$-free bismuth halide perovskites were exploited as solar absorbers. ${ }^{[24]}$ However, the optoelectronic properties of Bi-doped perovskites remain largely unexplored. In this work, we found that the PPC of the regular perovskite was surprisingly transformed into NPC after Bi doping. To the best of our knowledge, this is the first time NPC is reported in this type of hybrid materials. Our experimental results and first-principles calculations suggest the illumination-induced formation of DX-like defect centers, similar to doped III-V and chalcopyrite semiconductors. Trapping of photo-excited electrons at DX-like defects and subsequent recombination with mobile holes in the valence band underlies the observation of NPC in Bi-doped perovskites. Our results widen the horizon of perovskite research by intentional doping and defect engineering for tuning the optoelectronic properties of halide perovskites.

To exclude the influence of extrinsic factors such as grain boundaries on the transport properties, we focused our study on perovskite single crystals. Single crystals of undoped $\mathrm{MAPbBr} r_{3}$ and $\mathrm{Bi}$-doped $\mathrm{MAPbBr}_{3}$ were synthesized using the inverse temperature crystallization method as reported previously. ${ }^{[9 a, 25]}$ The crystal structure of Bi-doped $\mathrm{MAPbBr}_{3}$ is shown in Figure 1a. The actual amount of Bi content in the crystals is substantially lower than that of the precursor solution. For crystals grown from $1 \%$ Bi feed solution, the actual amount of Bi present is $0.012 \%$, which corresponds to $\sim 2 \times 10^{18} \mathrm{Bi}$ atoms per $\mathrm{cm}^{3}$ in the crystal. ${ }^{[9 a]} \mathrm{A}$ recent report confirmed that $\mathrm{Bi}$ doping create free electrons in $\mathrm{MAPbBr}_{3} .{ }^{[26]}$ 


\section{WILEY-VCH}

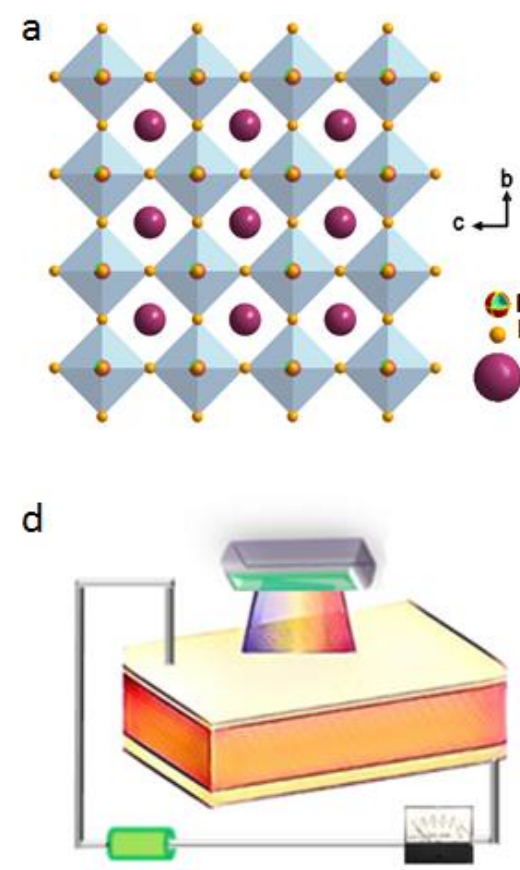

b
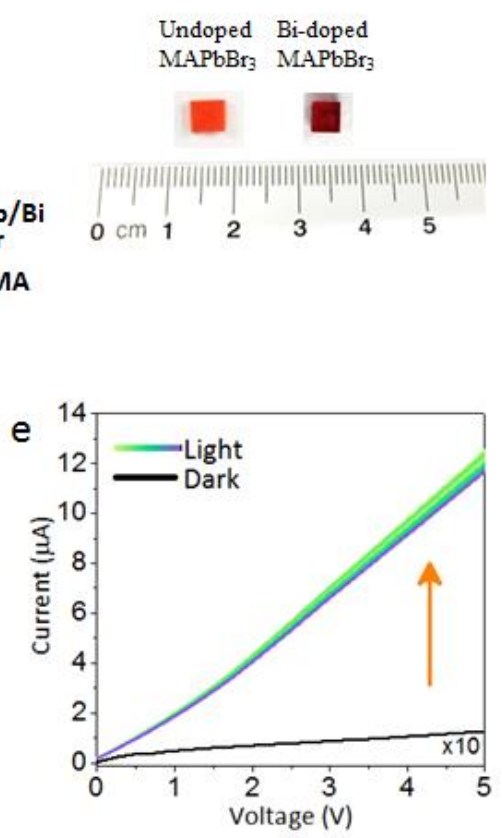

C
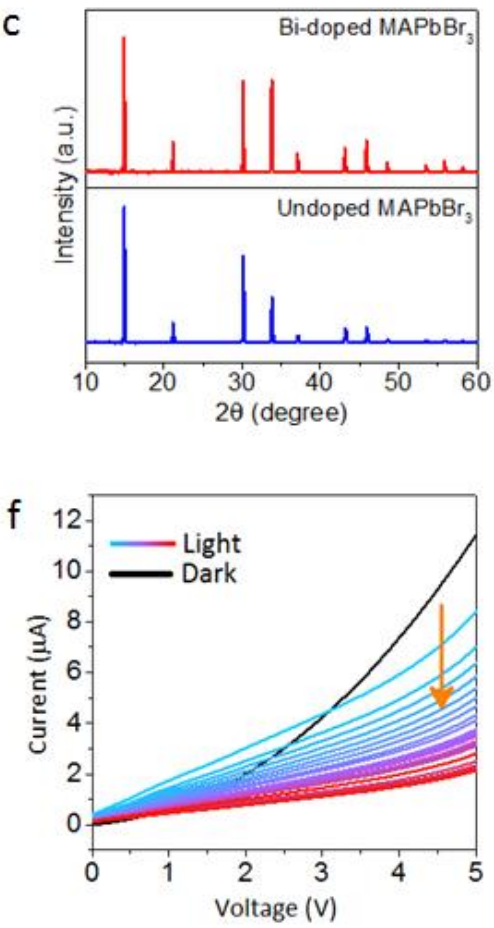

Figure 1. a) Schematic crystal structure of Bi-doped $\mathrm{MAPbBr}_{3}$. b) Photograph showing undoped and $\mathrm{Bi}$-doped $\mathrm{MAPbBr}_{3}$ crystals. c) XRD patterns of the crystals. d) Schematic device architecture used to study the photoconductance. Also shown are the current-voltage curves measured under white light $\left(5 \mathrm{~mW} / \mathrm{cm}^{2}\right)$ on e) undoped $\mathrm{MAPbBr}_{3}$ and f) $\mathrm{Bi}$-doped $\mathrm{MAPbBr}_{3}$. Measurements were started after initial light soaking of 3 mins. The measurement time for each $\mathrm{I}-\mathrm{V}$ curve is $20 \mathrm{~s}$ with an interval of $30 \mathrm{~s}$.

Figure $1 \mathrm{~b}$ shows the photo of undoped and $\mathrm{Bi}$-doped single crystals grown from $1 \% \mathrm{Bi}$ containing feed solution. Typically, the crystals have a lateral dimension of $4 \mathrm{~mm}$ and a thickness of $1 \mathrm{~mm}$. The undoped crystal has a bright orange color, in accordance to its bandgap of $2.2 \mathrm{eV}$, while the Bi-doped crystal exhibits a much dark color, consistent with previous reports. ${ }^{[9 a]}$ There is a red-shift in the absorption spectrum of the Bi-doped crystal with significant Urbach tail (Figure S1). These features can be attributed to the increase in the sub-bandgap density of states and lattice distortion as a result of Bi doping. ${ }^{[26-27]}$ The X-ray diffraction (XRD) pattern of powdered crystals of $\mathrm{Bi}$-doped $\mathrm{MAPbBr}_{3}$ does not exhibit any extra peaks compared to the undoped $\mathrm{MAPbBr}_{3}$ (Figure 1c), indicating a single-phase material with the intact perovskite structure. 


\section{WILEY-VCH}

To study the photoconductive behavior, we chose a simple device architecture with the single crystal being sandwiched between two Au electrodes (Figure 1d). The top Au electrode is approximately $25 \mathrm{~nm}$ thick and semitransparent. The dark current for the doped crystal was ten times higher than that of the undoped crystal. This enhancement in the dark current can be attributed to the n-type doping by Bi. To confirm this, we measured the work function (WF) by contact potential difference (CPD) and found that the WF for the Bi-doped crystal was $4.3 \mathrm{eV}$ in contrast to $4.5 \mathrm{eV}$ for the undoped $\mathrm{MAPbBr}_{3}$ crystal. As expected, for the undoped $\mathrm{MAPbBr}_{3}$, the sample current increases by almost two orders of magnitude upon light illumination (Figure 1e). The voltage sweep cycles were repeated for twenty times, and there was no sign of photo degradation. Surprisingly, when the same measurement is repeated for the Bi-doped crystals, there is an unusual decrease of photocurrent below the dark level, i.e. NPC, in the high bias region (Figure 1f). With each repetition of measurement, the current monotonously decreases and the region of NPC expands. In the 20th $I-V$ curve, the photocurrent decreased to a level of $2.8 \mu \mathrm{A}$ from $11 \mu \mathrm{A}$ (dark level) at a bias of $5 \mathrm{~V}$. The Bi doping appears to be very effective to trigger the photoresponse of perovskites to switch from positive to negative regime. In early works, NPC was generally observed under specific conditions such as low temperatures or with special sample architectures such as quantum wells. ${ }^{[28]}$

Measuring the transient photocurrent helps shed light on the mechanism behind the emergence of NPC in the Bi-doped perovskite. As shown in Figure 2a-b, both the undoped and Bi doped crystals exhibit a sharp increase of current when the light is switched on due to the quick light-induced generation of electron and hole pairs, which is followed by a slow decay of photocurrent. However, the photocurrent decay of the doped sample is much faster and more significant than the undoped counterpart, resulting in a bipolar behavior. Within $1.3 \mathrm{~s}$, the 


\section{WILEY-VCH}

photocurrent of the doped $\mathrm{MAPbBr}_{3}$ sample under illumination decreases below the dark level. Furthermore, when the illumination is switched off, the photocurrent of the undoped $\mathrm{MAPbBr}_{3}$ sample comes back to its dark level. In contrast, in the case of doped $\mathrm{MAPbBr}_{3}$, the dark current does not recover to its initial value, and instead a slow relaxation of current was observed.
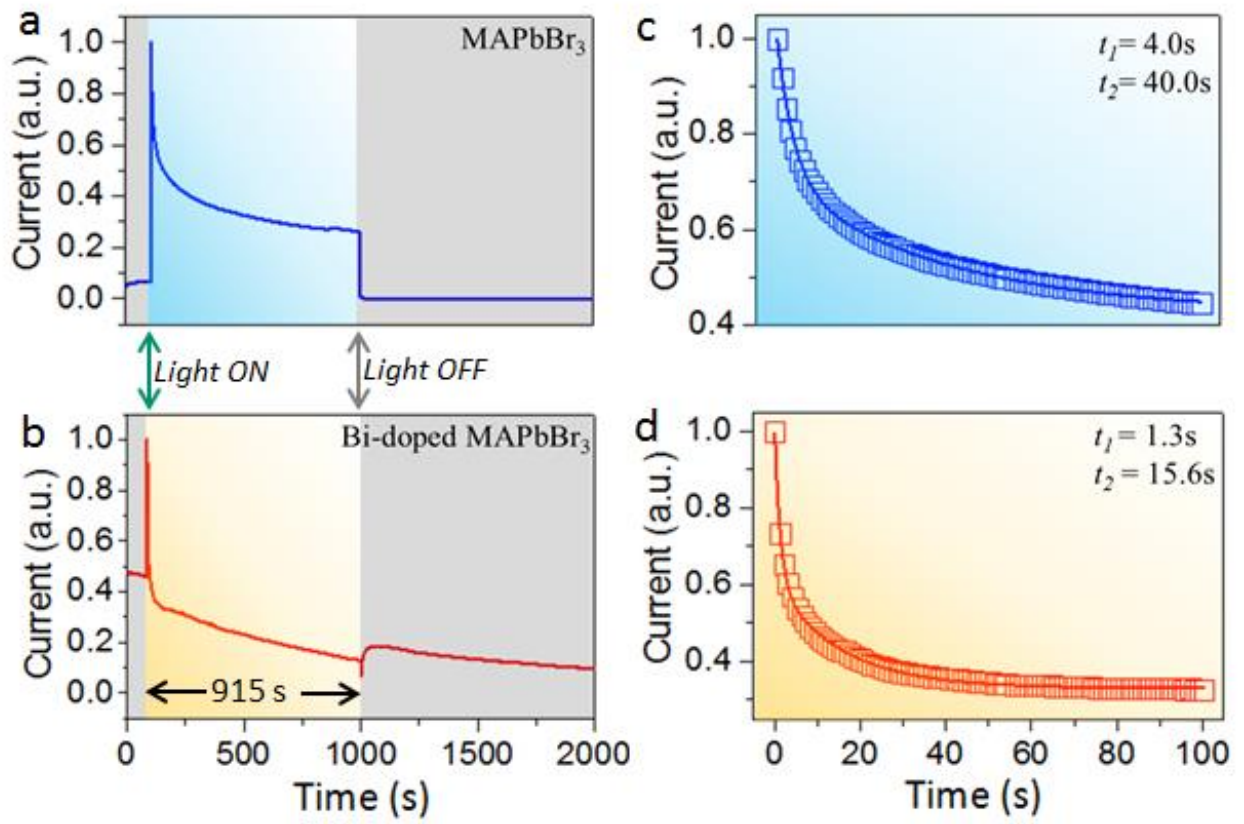

Figure 2. Transient photocurrent measured on undoped a) $\mathrm{MAPbBr}_{3}$ and b) Bi-doped $\mathrm{MAPbBr}_{3}$ samples. Gray regions indicate the condition when light is off. Photocurrent decay data of $\mathbf{c}$ ) undoped and d) doped $\mathrm{MAPbBr}_{3}$, along with the fitting curves. Light intensity is $5 \mathrm{~mW} / \mathrm{cm}^{2}$.

The fast decay right after the transient photocurrent enhancement in Figure 2a-b can be fitted with an exponential function with two time constants, i.e., $I=I_{0}+A_{1} e^{-t / t_{1}}+A_{2} e^{-t / t_{2}}$ (Figure 2c-d). The first smaller time constant $\left(t_{1}\right)$ is associated with trap-assisted recombination, and the second larger one $\left(t_{2}\right)$ with delayed charge trapping processes. ${ }^{[29]}$ Importantly, the time constant $t_{l}$ of the doped sample is three times smaller than that of the undoped sample, indicating that the charge recombination in the doped $\mathrm{MAPbBr}_{3}$ sample is significantly faster. For the doped sample, within $\sim 1.3$ seconds its photocurrent already goes below the dark level. Clearly, the trapassisted recombination is significantly accelerated by the Bi doping and serves as the main 


\section{WILEY-VCH}

mechanism underlying the NPC effect. In the previous works, ${ }^{[13,15,30]}$ the NPC is usually quite weak with a small photoconductivity yield $Y_{P C}=\left(I-I_{\text {dark }}\right) / I_{\text {dark }}$. In a recent work by Lee et al., ${ }^{[31]} \mathrm{a}$ large $Y_{\mathrm{PC}}$ of $34 \%$ was measured on graphene. In our case, Bi-doped $\mathrm{MAPbBr}_{3}$ presents the maximum $Y_{\mathrm{PC}}$ of $67 \%$, which is to our knowledge one of the largest effect reported so far. The interface between the Au electrode and the perovskite crystal can result in trapped charges. ${ }^{[32]}$ To confirm that the NPC effect is not due to trapped charges, sample geometry or due to device architecture, we fabricated a planar device with $\mathrm{Bi}$-doped $\mathrm{MAPbBr}_{3}$ microcystalline film and ITO electrodes. This planar device also exhibited the NPC effect proving its intrinsic nature (Figure S3).

It is already a consensus that $\mathrm{Bi}$ doping creates non-radiative recombination centers in perovskites deteriorating the optical properties. ${ }^{[26-27,33]}$ Our transient photocurrent measurements revealed the important role of trap-assisted recombination of photocarriers. The features in the transient photocurrent spectra in case of doped crystal are characteristics of NPC and have been observed in several prior works. ${ }^{[1,14,18,21 a, 34]}$ Although the importance of defects to the emergence of NPC was recognized, ${ }^{[17,35]}$ the nature of defects and their interaction with photocarriers have remained elusive so far. In hybrid perovskites, there have been conflicting reports on the defect characteristics, which could be a result of the variation of synthesis methods. Nevertheless, it is widely recognized that the defect levels in hybrid perovskites are shallow, giving rise to weak carrier trapping and excellent photovoltaic properties. ${ }^{[36]}$ However, such shallow defect levels are not expected to give rise to NPC. We hypothesize that the creation of DX-like centers (D: donor atom, X: unspecified lattice defect) in $\mathrm{MAPbBr}_{3}$ as a result of $\mathrm{Bi}$ doping and their activation upon optical excitation underlay the observation of NPC. Electrondonating impurities such as $\mathrm{Bi}_{\mathrm{Pb}}$ are usually expected to form shallow donor levels, but in certain 


\section{WILEY-VCH}

cases, shallow donors can convert into deep negatively charged DX-like centers after trapping electrons. ${ }^{[37]}$ One prerequisite of forming DX-like centers deep inside the bandgap is lattice distortion, which is consistent with the presence of the significant Urbach tail observed in the Bidoped $\mathrm{MAPbBr}_{3}{ }^{[26]}$ Although DX-like centers were reported as the major defect limiting the electron carrier density in zincblende or wurtzite semiconductors, ${ }^{[37-38]}$ they have not been proposed for any hybrid materials.

The proposed mechanism to explain the observed NPC is shown in Figure 3. Initially, shallow single donor levels are created just below the conduction band minima (CBM) as a result of $\mathrm{Bi}_{\mathrm{Pb}}(\alpha)$ doping (Figure 3a), and particularly the neutral substitutional donor $\mathrm{Bi}_{\mathrm{Pb}}$ can release electrons to the conduction band through thermal excitation, forming $\alpha^{+}$defect levels and producing free electrons. ${ }^{[37]}$ As shown in Figure 3b, upon light illumination, electrons are excited from the valence band ( $A$ in Figure $3 b$ ), and the holes are left behind ( $B$ in Figure $3 b$ ), which gives rise to the fast initial increase of photocurrent (Figure 3c shows the NPC curve corresponding to the transitions taking place relevant to DX-like center under light). The high density of the photoexcited electrons leads to a quasi-electron Fermi energy $\varepsilon_{f n}$ shifting closer to the CBM (Figure 3d), and after trapping the photogenerated electrons from the conduction band (C in Figure 3b), the DX-like center becomes energetically more stable than the substitutional donor defect. ${ }^{[38-39]}$ These trapped electrons in the DX-like center can recombine with holes from the valence band, leading to the fast decay of photocurrent ( $D$ in Figure 3c). When light is turned off, the photocurrent rapidly decreases ( $E$ in Figure 3c). However, without the illumination, the Fermi energy will move down towards its equilibrium value, and subsequently the DX-like center becomes less stable (Figure 3d) and will start to convert back into the shallow donor state, thus increasing the current ( $F$ in Figure 3c). 


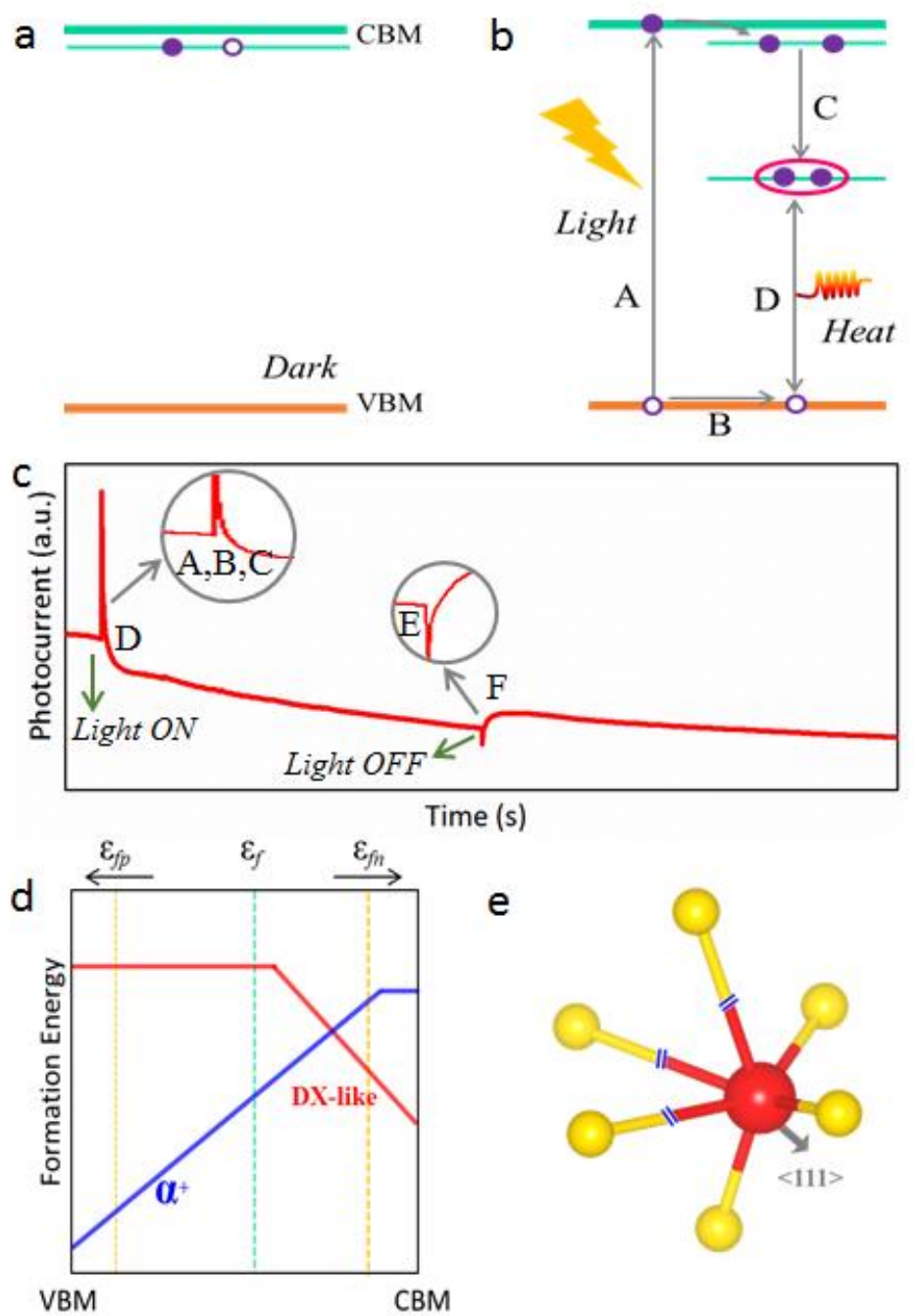

Figure 3. a) Schematic diagram of energy levels of the Bi-doped $\mathrm{MAPbBr}_{3}$ crystal in dark. b) Schematic representation of the emergence of DX-like defect levels under light illumination. Also shown are the dynamic photocarrier generation and recombination processes that lead to the NPC. c) NPC curve corresponding to the transitions taking place relevant to DX-like center under light. d) Schematic plot of formation energies of a shallow donor $\mathrm{Bi}_{\mathrm{Pb}}\left(\alpha^{+}\right)$and DX-like center in $\mathrm{MAPbBr}_{3}$ as functions of the Fermi level $\left(\varepsilon_{\mathrm{f}}\right)$. e) Atomic configuration of the DX-like center in $\mathrm{Bi}$-doped $\mathrm{MAPbBr}_{3}$.

Various forms of lattice distortion may occur to accompany the formation of DX-like centers. Our first-principle calculations show that when $\mathrm{Bi}$ replaces a $\mathrm{Pb}$ atom, which is six-fold coordinated with $\mathrm{Br}$ atoms in the lattice, $\mathrm{Bi}$ atom will displace along the $\langle 111\rangle$ direction, breaking three metal-halide bonds and resulting in the formation of a three-fold coordinated DX- 


\section{WILEY-VCH}

like (also denoted as DY) center (Figure 3e). It should be noted that the residual bond lengths are not equal due to the presence of MA cation. ${ }^{[40]}$ The DX-like center become stabilized because the electronic energy gain by transferring electrons from the shallow donor level to the deep DY level overcomes the energy cost of lattice distortion. More detailed description of the structure and energy levels inside the bandgap of the DY centers are discussed in a recent theoretical work. ${ }^{[0]}$ Recently, NPC was observed in some inorganic semiconductors and different mechanisms were proposed. In doped $\mathrm{MoS}_{2}$, photogenerated carriers interact with dopinginduced charges and form trions consisting of two electrons and one hole, and as a result, photoconductivity decreases due to increased carrier effective mass. ${ }^{[41]}$ In InAs nanowires, depletion of conduction channels by light-assisted hot electron trapping leads to the observation of NPC. ${ }^{[12]}$ Very recently, NPC was reported for Si-doped InGaN nanowires, which was attributed to carrier-carrier scattering owing to high carrier concentration. ${ }^{[42]}$

Since the carrier generation and recombination are energetic processes, ${ }^{[43]}$ one expects the results could have dependence on the illumination conditions and measurement temperature. Indeed, when UV light $\left(365 \mathrm{~nm}, 5 \mathrm{~mW} / \mathrm{cm}^{2}\right)$ or high-intensity white light $\left(20 \mathrm{~mW} / \mathrm{cm}^{2}\right)$ is employed, PPC was observed (Figure 4a), indicating that the carrier trapping at the DX centers is less effective under such strong lighting conditions. Furthermore, we found a strong temperature dependence of the photoconductivity: NPC appears only for T > $260 \mathrm{~K}$, whereas for $\mathrm{T}<260 \mathrm{~K}$, PPC was observed (Figure 4b). The temperature dependence suggests that there exists an energy barrier for the formation of DX-like centers, and the carrier trapping also becomes weaker at low temperatures (Figure 4c). In other words, the photocarrier generation rate dominates at low temperature and under high intensity light (or high photon energies). Also with decreasing temeprature, the bandgap of $\mathrm{MAPbBr}_{3}$ decreases ${ }^{[44]}$ which can also destabilize 
the DX-like centers. ${ }^{[40]}$ It should be noted that since doped crystals exhibit persistent photoconductivity, we followed a protocol reported previously to measure the temperature dependent resistance. ${ }^{[4]}$ The photoconductivity at each temperature was measured after heating the sample to room temperature, and the interval between measurements is more than one day to allow sufficient resistance relaxation. Finally, it is interesting to note that at $260 \mathrm{~K}$, the dark and light resistance is almost equal, giving rise to a negligible photoresponse (Figure 4d). Basically, NPC and PPC are competing phenomena, and a wide variety of photoresponse characteristics can be realized in $\mathrm{Bi}$-doped $\mathrm{MAPbBr}_{3}$ crystals.
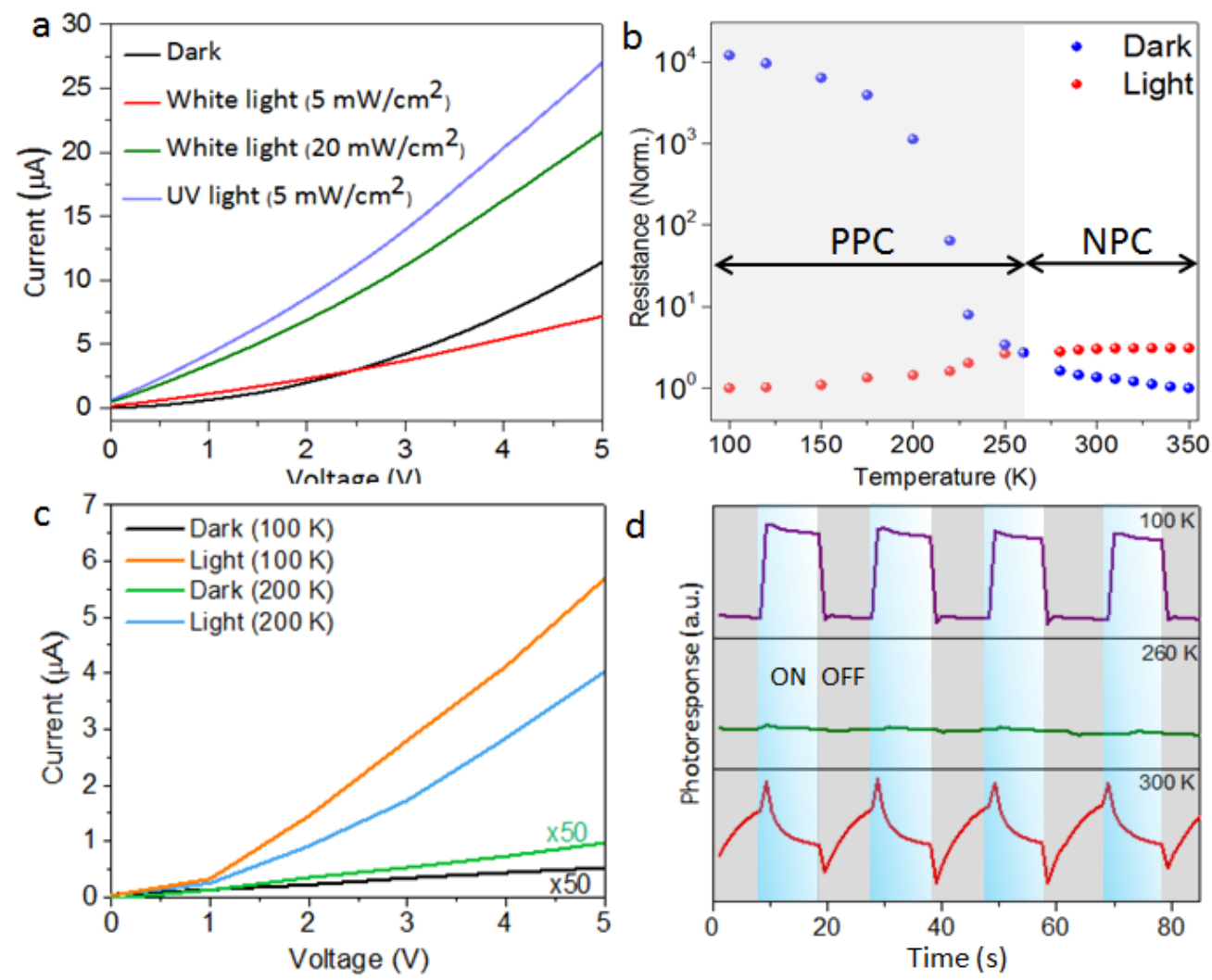

Figure 4. Light and temperature dependence of $\mathrm{Bi}$-doped $\mathrm{MAPbBr}_{3}$. a) I-V characteristics for the Bi-doped crystal showing PPC at room temperature under intense white light (20 $\mathrm{mW} / \mathrm{cm}^{2}$ ) and UV light. b) Resistance as a function of temperature measured either in dark or under light. Dark and light resistance data are normalized by their respective minimum values. c) $\mathrm{I}-\mathrm{V}$ characteristics for Bi-doped crystal at $200 \mathrm{~K}$ and $100 \mathrm{~K}$ under white light $\left(5 \mathrm{~mW} / \mathrm{cm}^{2}\right)$. d) Photoresponse of the Bi-doped crystal under pulsed illumination at three representative temperatures. 


\section{WILEY-VCH}

The formation of DX-like centers and the emergence of NPC also depend on the perovskite composition. Similar to $\mathrm{MAPbBr}_{3}$, Bi-doped $\mathrm{MAPbI}_{3}$ is single-phase and exhibits significant Urbach tail (Figure S3-S4). But, as shown in Figure S5, Bi-doped $\mathrm{MAPbI}_{3}$ shows PPC at room temperature. Furthermore, in $\mathrm{MAPbI}_{3}$, there is neither a spike-like surge of photocurrent when the light is switched on nor a fast decay of photocurrent as observed in $\mathrm{MAPbBr}_{3}$, which indicates much weaker carrier recombination in Bi-doped $\mathrm{MAPbI}_{3}$. Finally, we observed that doping-induced photoluminescence (PL) quenching in $\mathrm{MAPbI}_{3}$ (Figure S6) was significantly lower than that of $\mathrm{MAPbBr}_{3}$ (Figure S7), in line with the lower density of non-radiative recombination centers ${ }^{[27]}$ in $\mathrm{MAPbI}_{3}$. Our first-principles calculations indeed show that due to the small band gap of $\mathrm{MAPbI}_{3}, \mathrm{DX}$-like center is not stable in $\mathrm{MAPbI}_{3}$, consistent with experimental observations.

In summary, our work demonstrated that the light-matter interaction in hybrid perovskites can be tailored to a large degree via doping and forming DX-like centers. Particularly, Bi-doping at a low concentration is sufficient to induce NPC with a pronounced bipolar behavior in $\mathrm{MAPbBr}_{3}$ at room temperature, while only $\mathrm{PPC}$ exists in $\mathrm{MAPbI}_{3}$. Compared to conventional inorganic semiconductors, the DX-like defect centers in hybrid perovskites appear to be more susceptible to the factors like light illumination, temperature and composition. The discovery of NPC and DX-like centers highlights the unusual defect characteristics of hybrid perovskites, and our results indicate that defect engineering can be employed to tailor the optoelectronic properties of these solution-processed semiconductors.

\section{Supporting Information}

Supporting Information is available from the Wiley Online Library or from the author.

\section{Acknowledgement}

Research reported in this publication was supported by the King Abdullah University of Science and Technology (KAUST). D. Baran acknowledges the KAUST competitive research grant 


\section{WILEY-VCH}

(CRG7, \#3737) for financial support. The work at CSRC was supported by the National Key Research and Development Program of China under Grant No. 2016YFB0700700, the National Nature Science Foundation of China under Grant No. 51672023; 11634003; U1530401, and the Science Challenge Project, under Grant No. TZ20160003. M. A. H. and J. L. contributed equally to this work.

Received: ((will be filled in by the editorial staff))

Revised: ((will be filled in by the editorial staff))

Published online: ((will be filled in by the editorial staff))

\section{References}

[1] a) N.-G. Park, M. Grätzel, T. Miyasaka, K. Zhu, K. Emery, Nat. Energy 2016, 1, 16152; b) Yongbo Yuan, Tao Li, Qi Wang, Jie Xing, Alexei Gruverman, J. Huang, Sci. Adv. 2017, 3, e1602164; c) D. Bi, W. Tress, M. I. Dar, P. Gao, J. Luo, C. Renevier, K. Schenk, A. Abate, F. Giordano, J. P. Correa Baena, J. D. Decoppet, S. M. Zakeeruddin, M. K. Nazeeruddin, M. Gratzel, A. Hagfeldt, Sci. Adv. 2016, 2, e1501170; d) W. Nie, H. Tsai, R. Asadpour, J.-C. Blancon, A. J. Neukirch, G. Gupta, J. J. Crochet, M. Chhowalla, S. Tretiak, M. A. Alam, H.-L. Wang, A. D. Mohite, Science 2015, 347, 522; e) W. Chen, Y. Wu, Y. Yue, J. Liu, W. Zhang, X. Yang, H. Chen, E. Bi, I. Ashraful, M. Gratzel, L. Han, Science 2015, 350, 944; f) J. H. Heo, D. H. Song, S. H. Im, Adv. Mater. 2014, 26, 8179; g) W. J. Yin, T. Shi, Y. Yan, Adv. Mater. 2014, 26, 4653.

[2] C. K. Moller, Nature 1958, 182, 1436.

[3] a) S. D. Stranks, H. J. Snaith, Nat Nano 2015, 10, 391; b) M. I. Saidaminov, M. A. Haque, M. Savoie, A. L. Abdelhady, N. Cho, I. Dursun, U. Buttner, E. Alarousu, T. Wu, O. M. Bakr, Adv. Mater. 2016, 28, 8144; c) Y.-H. Kim, H. Cho, J. H. Heo, T.-S. Kim, N. Myoung, C.-L. Lee, S. H. Im, T.-W. Lee, Adv. Mater. 2015, 27, 1248.

[4] W. Wei, Y. Zhang, Q. Xu, H. Wei, Y. Fang, Q. Wang, Y. Deng, T. Li, A. Gruverman, L. Cao, J. Huang, Nat. Photonics 2017, 11, 315.

[5] S. Yakunin, D. N. Dirin, Y. Shynkarenko, V. Morad, I. Cherniukh, O. Nazarenko, D. Kreil, T. Nauser, M. V. Kovalenko, Nat. Photonics 2016, 10, 585.

[6] a) A. Fu, P. Yang, Nat. Mater. 2015, 14, 557; b) H. Zhu, Y. Fu, F. Meng, X. Wu, Z. Gong, Q.

Ding, M. V. Gustafsson, M. T. Trinh, S. Jin, X. Y. Zhu, Nat. Mater. 2015, 14, 636.

[7] C. Gu, J.-S. Lee, ACS Nano 2016, 10, 5413.

[8] S.-T. Ha, C. Shen, J. Zhang, Q. Xiong, Nat. Photonics 2015, 10, 115.

[9] a) A. L. Abdelhady, M. I. Saidaminov, B. Murali, V. Adinolfi, O. Voznyy, K. Katsiev, E. Alarousu, R. Comin, I. Dursun, L. Sinatra, E. H. Sargent, O. F. Mohammed, O. M. Bakr, J. Phys. Chem. Lett. 2016, 7, 295; b) S. T. Williams, A. Rajagopal, S. B. Jo, C. C. Chueh, T. F. L. Tang, A. Kraeger, A. K. Y. Jen, J. Mater. Chem. A 2017, 5, 10640; c) S. Shahbazi, C.-M. Tsai, S. Narra, C.-Y. Wang, H.-S. Shiu, S. Afshar, N. Taghavinia, E. W.-G. Diau, J. Phys. Chem. C 2017, 121, 3673; d) J. Navas, A. Sánchez-Coronilla, J. J. Gallardo, J. C. Piñero, D. De los Santos, E. I. Martín, N. C. Hernández, R. Alcántara, C. Fernández-Lorenzo, J. Martín-Calleja, Nano Energy 2017, 34, 141.

[10] M. C. P. Chang, C. M. Penchina, J. S. Moore, Phy. Rev. B 1971, 4, 1229.

[11]J. Dresner, J. Chem. Phys. 1961, 35, 1628. 


\section{WILEY-VCH}

[12] Y. Yang, X. Peng, H. S. Kim, T. Kim, S. Jeon, H. K. Kang, W. Choi, J. Song, Y. J. Doh, D. Yu, Nano Lett. 2015, 15, 5875.

[13] H. Nakanishi, K. J. Bishop, B. Kowalczyk, A. Nitzan, E. A. Weiss, K. V. Tretiakov, M. M. Apodaca, R. Klajn, J. F. Stoddart, B. A. Grzybowski, Nature 2009, 460, 371.

[14] R. Sreekumar, R. Jayakrishnan, C. Sudha Kartha, K. P. Vijayakumar, J. Appl. Phys. 2006, $100,033707$.

[15] J.-L. Zhu, G. Zhang, J. Wei, J.-L. Sun, Appl. Phys. Lett. 2012, 101, 123117.

[16] X. Zhang, J. Jie, Z. Wang, C. Wu, L. Wang, Q. Peng, Y. Yu, P. Jiang, C. Xie, J. Mater. Chem. 2011, 21, 6736.

[17] F. Stockmann, Z. Physik 1955, 143, 348.

[18] P.-C. Wei, S. Chattopadhyay, M.-D. Yang, S.-C. Tong, J.-L. Shen, C.-Y. Lu, H.-C. Shih, L.C. Chen, K.-H. Chen, Phys. Rev. B 2010, 81, 045306.

[19]B. Borshchevskii, J. Phys. Chem. USSR 1947, 21, 1007.

[20] E. G. Miselyuk, E. B. Mertens, Izvest. Akad. Nauk SSSR Ser. Fiz. 1952, 16, 115.

[21] a) A. Serpi, Phys. Stat. Sol. A 1992, 133, K73; b) N. Gogurla, A. K. Sinha, D. Naskar, S. C. Kundu, S. K. Ray, Nanoscale 2016, 8, 7695.

[22] A. G. Aleksanyan, Sov. J. Quant. Electron 1975, 4, 933.

[23] a) Y. Zhou, Z. J. Yong, K. C. Zhang, B. M. Liu, Z. W. Wang, J. S. Hou, Y. Z. Fang, Y. Zhou, H. T. Sun, B. Song, J. Phys. Chem. Lett. 2016, 7, 2735; b) Y. Zhou, Z.-J. Yong, W. Zhang, J.-P. Ma, A. Sadhanala, Y.-M. Chen, B.-M. Liu, Y. Zhou, B. Song, H.-T. Sun, J. Mater. Chem. C 2017, 5, 2591.

[24] B.-W. Park, B. Philippe, X. Zhang, H. Rensmo, G. Boschloo, E. M. J. Johansson, Adv. Mater. 2015, 27, 6806.

[25] G. Maculan, A. D. Sheikh, A. L. Abdelhady, M. I. Saidaminov, M. A. Haque, B. Murali, E. Alarousu, O. F. Mohammed, T. Wu, O. M. Bakr, J. Phys. Chem. Lett. 2015, 6, 3781.

[26] Y. Yamada, M. Hoyano, R. Akashi, K. Oto, Y. Kanemitsu, J. Phys. Chem. Lett. 2017, 8, 5798.

[27] P. K. Nayak, M. Sendner, B. Wenger, Z. Wang, K. Sharma, A. J. Ramadan, R. Lovrincic, A. Pucci, P. K. Madhu, H. J. Snaith, J. Am. Chem. Soc. 2018, 140, 574.

[28] a) R. A. Höpfel, Appl. Phys. Lett. 1988, 52, 801; b) A. S. Chaves, H. Chacham, Appl. Phys. Lett. 1995, 66, 727.

[29] G. Gordillo, C. A. Otalora, A. A. Ramirez, Phys. Chem. Chem. Phys. 2016, 18, 32862.

[30] H. Zhang, J. Yao, J. Shao, H. Li, S. Li, D. Bao, C. Wang, G. Yang, Sci. Rep. 2014, 4, 5876.

[31] C. Biswas, F. Gunes, D. L. Duong, S. C. Lim, M. S. Jeong, D. Pribat, Y. H. Lee, Nano Lett. 2011, $11,4682$.

[32] Y. Rakita, E. Meirzadeh, T. Bendikov, V. Kalchenko, I. Lubomirsky, G. Hodes, D. Ehre, D. Cahen, APL Materials 2016, 4, 051101.

[33] a) R. Meng, G. Wu, J. Zhou, H. Zhou, H. Fang, M. A. Loi, Y. Zhang, Chemistry 2019, 25, 5480; b) O. A. Lozhkina, A. A. Murashkina, V. V. Shilovskikh, Y. V. Kapitonov, V. K. Ryabchuk, A. V. Emeline, T. Miyasaka, J. Phys. Chem. Lett. 2018, 9, 5408.

[34] C. M. Penchina, J. S. Moore, N. Holonyak, Phys. Rev. 1966, 143, 634.

[35] R. H. Bube, Photoconductivity of Solids. John Wiley \& Sons: 1960.

[36] a) C. Eames, J. M. Frost, P. R. Barnes, B. C. O'Regan, A. Walsh, M. S. Islam, Nat. Commun. 2015, 6, 7497; b) Q. Chen, H. Zhou, Y. Fang, A. Z. Stieg, T. B. Song, H. H. Wang, X. Xu, Y. Liu, S. Lu, J. You, P. Sun, J. McKay, M. S. Goorsky, Y. Yang, Nat. Commun. 2015, 6, 7269; c) Z. Chu, M. Yang, P. Schulz, D. Wu, X. Ma, E. Seifert, L. Sun, X. Li, K. Zhu, K. Lai, 


\section{WILEY-VCH}

Nat. Commun. 2017, 8, 2230; d) C. Motta, F. El-Mellouhi, S. Kais, N. Tabet, F. Alharbi, S. Sanvito, Nat. Commun. 2015, 6, 7026.

[37] S. Lany, A. Zunger, Phys. Rev. Lett. 2008, 100, 016401.

[38] D. J. Chadi, K. J. Chang, Phys. Rev. B 1989, 39, 10063.

[39] a) Hans J. Queisser, E. E. Haller, Science 1998, 281, 945; b) A. Oshiyama, S. Ohnishi, Phys. Rev. B 1986, 33, 4320.

[40] J.-L. Li, J. Yang, T. Wu, S.-H. Wei, J. Mater. Chem. C 2019, 7, 4230.

[41] C. H. Lui, A. J. Frenzel, D. V. Pilon, Y. H. Lee, X. Ling, G. M. Akselrod, J. Kong, N. Gedik, Phys. Rev. Lett. 2014, 113, 166801.

[42] R. Bose, A. Adhikari, V. M. Burlakov, G. Liu, M. A. Haque, D. Priante, M. N. Hedhili, N. Wehbe, C. Zhao, H. Yang, T. K. Ng, A. Goriely, O. M. Bakr, T. Wu, B. S. Ooi, O. F. Mohammed, ACS Energy Lett. 2018, 3, 476.

[43] a) M. A. B. Tavares, M. J. da Silva, M. L. Peres, S. de Castro, D. A. W. Soares, A. K. Okazaki, C. I. Fornari, P. H. O. Rappl, E. Abramof, Appl. Phys. Lett. 2017, 110, 042102; b) E. Gombia, R. Mosca, S. Franchi, C. Ghezzi, R. Magnanini, J. Appl. Phys. 1998, 84, 5337.

[44] a) J. Dai, H. Zheng, C. Zhu, J. Lu, C. Xu, J. Mater. Chem. C 2016, 4, 4408; b) A. M. Soufiani, F. Huang, P. Reece, R. Sheng, A. Ho-Baillie, M. A. Green, Appl. Phys. Lett. 2015, 107, 231902.

[45] M. J. P. Pirralho, M. L. Peres, D. A. W. Soares, P. C. O. Braga, F. S. Pena, C. I. Fornari, P. H. O. Rappl, E. Abramof, Phys. Rev. B 2017, 95, 075202. 


\section{WILEY-VCH}

Positive photoconductivity in $\mathrm{CH}_{3} \mathrm{NH}_{3} \mathrm{PbBr}_{3}$ turns into negative photoconductivity after $\mathrm{Bi}$ doping. This transition is due to photogenerated DX-like centers in Bi-doped $\mathrm{CH}_{3} \mathrm{NH}_{3} \mathrm{PbBr}_{3}$.

Keyword: Hybrid perovskites, doping, negative photoconductance, DX centers

Md Azimul Haque, Jin-Ling Li, Ahmed L. Abdelhady, Makhsud I. Saidaminov, Derya Baran, Osman M. Bakr, Su-Huai Wei*, Tom Wu*

Transition from Positive to Negative Photoconductance in Doped Hybrid Perovskite Semiconductors

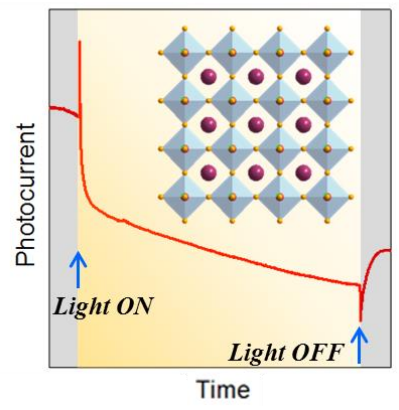

